\title{
A discrete-trials PREE in an operant situation*
}

\author{
STEPHEN C. BITGOOD and JOHN R. PLATT \\ University of Iowa, Iowa City, Iowa 52240
}

Three groups of pigeons under different ratio schedules of reinforcement received acquisition and extinction training with a discrete-trials operant procedure. A group with all trials reinforced was less resistant than a $50 \%$ reinforcement group. Any increments in resistance to extinction due to variations in number of responses per food presentation were demonstrated to be inadequate to explain this discrete-trials partial-reinforcement extinction effect. It was concluded that discrete-trials resistance to extinction is more sensitive to acquisition variations in reinforcement per trial than in reinforcement per response.

Two distinguishable types of paradigms are commonly employed to study instrumental conditioning: the discrete-trials runway and the free-responding operant chamber. The discrete-trials situation is typified by $E$ control over the trial units and the free-operant situation by the lack of this restriction. Free-operant situations have often been converted to discrete-trials situations by introducing a blackout (timeout) between trials.

The partial-reinforcement extinction effect (PREE) has been observed in both free-operant and discrete-trials paradigms. The PREE refers to the observation that measures of resistance to extinction are increased if reinforcements have been inconsistently administered during acquisition training. Although discrete-trials and free-operant partial reinforcement procedures both yield greater resistance to extinction than corresponding continuous reinforcement procedures, several authors have suggested that different variables may be involved. For example, Platt (in press) observed that free-operant studies have typically manipulated the response requirement for reinforcement, while discrete-trials studies have dealt with variations in the probability of reinforcement per trial. That is, free-operant procedures have varied the response-chain length per reinforcement, e.g., fixed ratio (FR) 5 vs FR 1, while discrete-trials procedures have held the runway length constant and varied the frequency of reinforcement of trials. Examples of free-operant PREE investigations are Mowrer \& Jones (1945) and Denny, Wells, \& Maatsch (1957), both of which found that larger FRs in acquisition produced more responses in free-operant extinction. On the other hand, Porter \& Hug (1965) and Gonzales, Bainbridge, \& Bitterman (1966)

* The present research was supported by United State Public Health Service Grant MH-15380, John R. Platt, principle investigator. This report is based on part of a Master's thesis completed by the first author and submitted to the University of Iowa Graduate School. utilized discrete-trials operant procedures to study resistance to extinction with the length of the response ratio or trial equated and reinforcement frequency for trials varied. Both studies found that partially reinforced Ss were more resistant to extinction than continuously reinforced ones.

Platt (in press) reported an experiment using second-order reinforcement schedules (Kelleher, 1966) to demonstrate the usual discrete-trials PREE with an operant procedure, while controlling for free-operant-like PREE. This experiment attempted to test the hypothesis that discrete-trials extinction is mainly sensitive to the probability of reinforcement per discrete trial rather than the probability of reinforcement per response. Three groups of pigeons received 18 sessions of discrete-trials acquisition followed by a discrete-trials extinction procedure in an operant keypecking chamber. The continuous group received reinforcement for each FR 30 trial. The partial group received random $50 \%$ reinforcement of its FR 30 trials. Finally, an intermittent group received $100 \%$ reinforcement of variable-ratio (VR) trials with a mean response requirement of 60 . This group was a control for free-operant PREE effects in discrete-trials extinction in that its acquisition trials were continuously reinforced, but reinforcement was received after the same numbers of responses as in the partial group. This study found greatest resistance to extinction for the partial group, but the effect was not as striking as expected. It was felt that more power was needed to consider this paradigm experimentally worth while.

One possible reason for lack of more substantial performance differences in the experiment reported by Platt was that the common pretraining procedure may have attenuated differential schedule effects. Pretraining is necessary in this operant paradigm in order to obtain the desired response. A "naive" pigeon does not initially peck a stimulus key 30 or more times without reinforcement. The operant keypecking situation is to be contrasted with the runway paradigm where experimental conditions can commence with the first placement of the animal into the apparatus.

Since pretraining may have increased resistance to extinction for the continuous schedule used by Platt, a procedure was sought that would allow evaluation of this effect. It was reasoned that attenuation of the effects of pretraining on acquisition and extinction performance might be accomplished by the use of different colored stimulus keys in order to minimize generalization from pretraining. The present experiment attempted to determine the effects of pretraining on subsequent acquisition and the PREE by comparing Ss that were shifted to another stimulus in acquisition and extinction with ones that continued to receive the pretrained stimulus.

\section{SUBJECTS}

Twenty-four naive male homing pigeons, 6 to 18 months of age, were maintained at $80 \%$ of their free-feeding weights.

\section{APPARATUS}

Four single-key operant chambers were used. The chambers were similar to that described by Ferster \& Skinner (1957). Reinforcement was 3-sec access to Purina Pigeon Checkers in a Lehigh Valley Electronics food hopper. The houselight was a small pilot lamp that was illuminated whenever the key light or hopper light was activated. Automatic scheduling and storage of data were accomplished simultaneously in all four chambers by a Digital Equipment Corporation PDP-8/I computer, and data was recorded by an on-line Teletype.

\section{PROCEDURE}

The birds were autoshaped to peck a key according to the procedure described by Brown \& Jenkins (1968). After the birds were pecking the key reliably, they received two sessions of exposure to a procedure in which the response ratio was gradually increased from 1 to 30 keypecks. Some birds required an extra day of this procedure because of difficulty in maintaining responding when the ratio was increased. One-half of the Ss were autoshaped and pretrained with a red key and the other half with a green key. After pretraining, each color group was divided into two subgroups, a nonshifted group which received the pretrained color throughout acquisition and extinction and a shifted group which received the other stimulus color. These shifted and nonshifted groups were further divided according to schedule of reinforcement. The continuous group 
Table 1

Acquisition Start Speeds

\begin{tabular}{lcc} 
& Nonshift & Shift \\
\hline Continuous & .317 & .712 \\
Partial & .536 & .441 \\
Intermittent & .346 & .187 \\
\hline
\end{tabular}

Table 2

Extinction Ratio Rate Transformations

\begin{tabular}{lcc}
\hline & Red & Green \\
\hline Nonshift & .394 & .362 \\
Shift & .221 & .588 \\
\hline
\end{tabular}

received food and the hopper light, followed by blackout, after every trial of 30 pecks. In the second-orderschedule notation of Kelleher (1966), this condition would be denoted CRF(FR30:S, TO). The partial group was fed after a random one-half of its 30 -response trials, i.e. VR2(FR30:S, TO). The sequence of reinforced and nonreinforced trials was determined by Gellerman's (1933) series. Nonreinforced trials terminated with presentation of the hopper light only, while on reinforced trials the hopper light was accompanied by presentation of food. The intermittent group received reinforcement after a variable number of responses that averaged 60 , i.e., CRF(VR60:S, TO). This group received reinforcement after the same number of responses as the partial group but had no distinctively nonreinforced trials. A 20-sec timeout (blackout) was programmed to follow the offset of every hopper light for all groups. After 24 sessions of acquisition training, consisting of 24 trials each for the continuous and partial groups and of 12 trials for the intermittent group, extinction training began. During

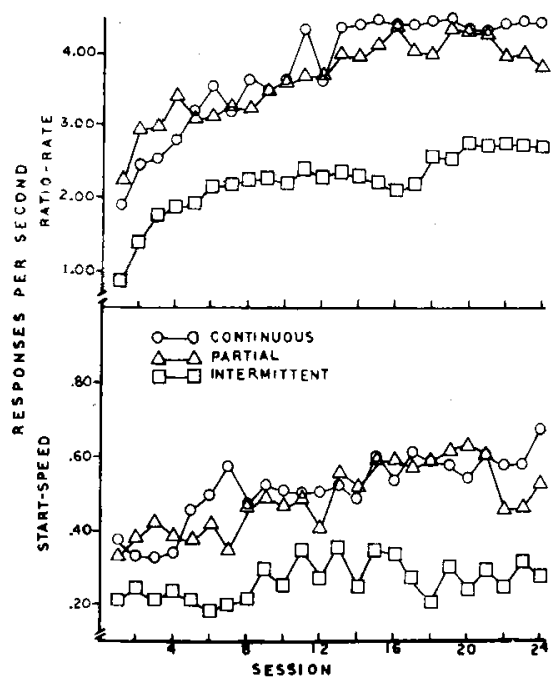

Fig. 1. Mean ratio rates and start speeds across acquisition sessions for each of the three reinforcement schedules. extinction, all groups were treated identically. Thirty responses turned off the key light and illuminated the hopper light for $3 \mathrm{sec}$, without food being presented. The extinction procedure would thus be denoted EXT(FR30:S, TO). An arbitrary time limit of $120 \mathrm{sec}$ without completion of the 30 responses terminated a trial with the presentation of the hopper light alone. All hopper lights continued to be followed by a 20 -sec blackout. All groups received 24 trials per extinction session. Ss were considered extinguished when they failed to make a response on 20 consecutive trials.

\section{RESULTS}

One bird each in the partial and continuous groups was replaced, one because of extremely long latencies in acquisition and the other because, on the first session of extinction, responses occurred on only 3 of 24 trials. The bird with long latencies would not respond during extinction because of the 120 -sec time limit on a trial. The other bird responded on substantially fewer trials (8) than any other bird in the experiment. It was felt that this extremely aberrant performance should not be included in the analysis.

The latency of each S's first response of each trial was reciprocalized and averaged for each session to form a start-speed measure. Similarly, the mean number of responses per second, excluding the time for the first response, was computed for each $\mathrm{S}$ on each trial to obtain a ratio-rate measure.

Acquisition

The effects of sessions, schedules, shift, and color were analyzed by means of separate analyses of variance for ratio rates and start speeds. Ratio rates were an increasing function of sessions in a cquisition, $\mathrm{F}(23,276)=12.79, \quad \mathrm{p}<.001$. The schedule effects, graphed across sessions in the upper frame of Fig. 1, a p proached significance, $F(2,12)=3.32, p=.071$. The overall means were $3.81,3.67$, and 2.22 responses per second for the continuous, partial, and intermittent groups, respectively. The only other statistically reliable factor was color, with the green stimulus resulting in higher rates $(3.85)$ than the red (2.61), $F(1,12)=4.87, p<.05$.

Start speeds were also an increasing function of sessions in acquisition, $F(23,276)=4.36, \quad p<.001, \quad$ and yielded a significant schedule effect, $F(2,12)=3.92, \quad p<.05$. Start-speed means for each schedule are plotted across sessions in the lower frame of Fig. 1. It is apparent from examination of the figure that the intermittent Ss were again slower than the other groups. The color main effect also effect also approached significance, $F(1,12)=3.60, p=.082$, with green (.499) again faster than red (.348). In addition to these simple effects in the start-speed measures, several interactions were found. The means in Table 1 reflect a significant Schedules by Shift interaction, $F(2,12)=4.84$, $\mathrm{p}<.05$, with shifted Ss showing larger differences between schedules than nonshifted Ss. The interaction of color with shift approached significance, $F(1,12)=3.75, p=.08$, and increased across sessions so as to produce a significant Color by Shift by Sessions interaction, $F(23,276)=2.74$, $\mathrm{p}<.001$. These latter two interactions reflected a tendency for color to affect start speeds only in the shifted condition. Thus, mean acquisition start speeds were .40 responses per second for both red and green when the pretrained color was used in acquisition. However, when the color was shifted from pretraining to acquisition, the means were .60 for green and .30 for red.

\section{Extinction}

Since the asymptotic levels of acquisition ratio rates and start speeds indicated performance differences due to color and schedule, as well as several interactions, Anderson (1963) transforms were calculated for each session of extinction

'I'ranstormed ratio rates were a decreasing function of sessions, $F(6,72)=63.06, p<.001$. The schedule effect was also reliable, $F(2,12)=12.94, p<.001$, with the overall means being .287, .596, and .292 for continuous, partial, and intermittent, respectively. Transformed ratio rates for the three schedules are plotted across extinction sessions in Fig. 2. The partial group was clearly more resistant to extinction than either the continuous or intermittent group. The color effect was also significant, $F(1,12)=8.68$, $\mathrm{p}<.05$. As in acquisition, green resulted in higher extinction performance $(.475)$ than red (.308). Finally, color was found to interact with shift, $F(1,12)=12.72, p<.01$. The extinction means averaged over sessions for the combinations of color and shift are shown in Table 2. Shifring to red resulted in more rapid extinction than always having been on red, but shifting to green was superior to always having been on green. None of these color or shift effects interacted with schedule. In particular, the expected Schedule by Shift interaction was not statistically reliable, $\quad F(2,12)=2.20, \quad \mathrm{p}>.10$. Extinction start speeds are not presented here since Anderson transforms of them yielded sessions as the only significant effect, $F(6,72)=53.45, p<.001)$. 


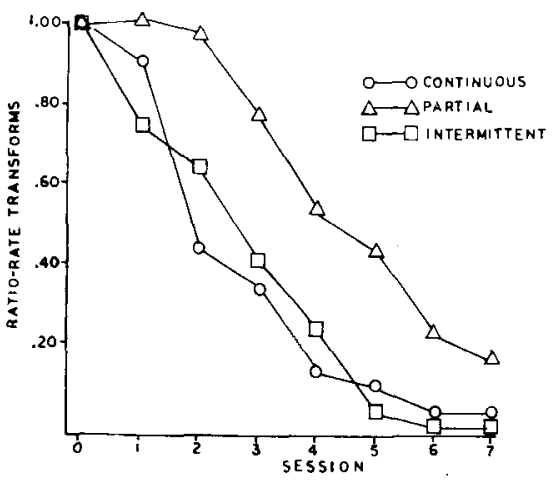

Fig. 2. Mean Anderson transformations of ratio rates in extinction for each of the three acquisition reinforcement schedules.

\section{DISCUSSION}

The partial and intermittent groups were observed to respond differentially in acquisition, despite the fact that both schedules were reinforced after the same number of responses and received the same number of reinforcements per session. The basis of the differential responding between these two groups was probably conditioned reinforcement. The partial group received a hopper light without presentation of the food hopper on nonreinforced trials. Since food presentation was always accompanied by the hopper light, it is reasonable to assume that the hopper light possessed conditioned reinforcing value. Thus, the partial Ss received some reinforcement, either primery or conditioned, after every $\mathbf{3 0}$ keypecks. The intermittent Ss did not receive this conditioned reinforcing stimulus after every 30 keypecks, but were required to complete from 30 to 120 responses for reinforcement.

Another consistent effect in both start speeds and ratio rates during acquisition was faster responding to the green key than to the red key, particularly when key color was changed from pretraining to acquisition. It was suspected that off-key pecking may have been responsible for this color effect since off-key pecking is known to occur (e.g., Bachrach, 1966) and may be a function of the properties of the stimulus. To test this interpretation, four bixds from the experiment were retrained, two previously trained and extinguished on red and two on green. One bird of each color had received the partial schedule and the other the continuous one. The birds were retrained with their acquisitionextinction color on the continuous schedule, and off-key pecks were recorded on mimeograph masters which were taped to the wall of the chamber with a circle cut out to expose the response key. For both red-key birds, the mimeograph masters were consistently blackened with off-key pecks, either immediately above or below the key. Records for the green-key birds, on the other hand, never showed more than a few discrete impressions from off-key pecks. It remains to be seen if this greater amount of off-key pecking, and-hence lower response rates to the red key, was the result of some unique property of the present operant chambers.

The only evidence obtained for the masking of schedule effects by the common pretraining procedure was the fact that shifted stimulus birds demonstrated a start-speed schedule effect in acquisition (continuous, partial, and intermittent speeds in decreasing order), whereas nonshifted birds, as in the experiment reported by Platt (in press), showed little difference between schedules in acquisition. No interactions between schedule and shift were found in extinction. In spite of the failure to demonstrate larger acquisition schedule effects on resistance to extinction when key color was changed from pretraining to acquisition, very large overall effects of acquisition schedule on resistance to extinction of ratio rates were found. In particular, the partial group was much more resistant to extinction than the continuous and intermittent groups, which in turn did not differ from each other. This result supports the original hypothesis that discrete-trials FR extinction is very sensitive to reinforcement per discrete trial, but relatively insensitive to probability of reinforcement per individual response. Logan \& Wagner (1965, pp. 56-57) have proposed that an operant response is analogous to taking a step in a discrete-trials runway. Thus, a VR reinforcement schedule was not seen as a partial reinforcement procedure in the same sense as partial reinforcement in the runway since the former varies the number of responses in a "trial" rather than the probability of reinforcement of the trial. These authors proposed a study comparing $50 \%$ partial reinforcement in a 1 - $\mathrm{ft}$ fixed-distance runway with continuous reinforcement in a variable-distance runway with a mean of $2 \mathrm{ft}$. The fixed-distance condition represented partial reinforcement in the discrete-trials sense, and the variable-distance condition represented partial reinforcement in the usual free-operant sense. Logan \& Wagner predicted that the fixed-distance condition would produce greater resistance to extinction for a discrete-trials extinction procedure, but that the variable-distance Ss would run farther in an endless runway (totally free-operant extinction). The present results support the first half of this prediction, but it remains to be seen if totally free-operant extinction is more sensitive to reinforcement per individual response than to reinforcement per discrete trial. The present results also leave unanswered the question of whether the timeouts or intertrial intervals used here were necessary to obtaining discrete-trials-like results for resistance to extinction, or if any brief stimulus change presented on the same contingencies would have had similar effects. If the latter proves to be the case, then the distinction between free-operant and discrete-trials paradigms becomes trivial indeed, in the sense that any discrete-trials procedure could be simulated while still allowing totally free responding (Platt, in press).

\section{REFERENCES}

ANDERSON, N. G. Comparison of different populations: Resistance to extinction and transfer. Psy chological Review, 1963, 70, 162-179.

BACHRACH, A. J. A simple method of obtaining a scatter distribution of off-key pigeon pecking. Journal of the Experimental Analysis of Behavior, 1966, $9,152$.

BROWN, P. L., \& JENKINS, H. $M$ Auto-shaping of the pigeon's key-peck. Journal of the Experimental Analysis of Behavior, 1968, 11, 1-8.

DENNY, M, R., WELLS, R. H., \& MAATSCH, J. L. Resistance to extinction as a function of the discrimination habit established during fixed-ratio reinforcement. Journal of Experimental Psychology, 1957, 6, 451-456.

FERSTER, C. B. \& SKINNER, B. F Schedules of reinforcement. New York: Appleton-Century-Crofts, 1957.

GELLERMAN, L. W. Chance orders of alternating stimuli in visual discrimination experiments. Journal of Genetic Psychology, 1933, 42, 206-208.

GONZALES, R. C., BAINBRIDGE, P., \& BITTERMAN, M. E. Discrete-trials lever pressing in the rat as a function of pattern of reinforcement, effortfulness of response, and amount of reward. Journal of Comparative \& Physiological Psychology, 1966, 61, 110-122.

KELLEHER R T Conditioned reinforcement in second-order schedules. Journal of the Experimental Analysis of Behavior, $1966,9,475-485$

LOGAN, F. A., \& WAGNER, A. R, Reward and punishment. Boston: Allyn \& Bacon, 1965.

MOWRER, O. H., \& JONES, H. M. Habit strength as a function of the pattern of reinforcement. Journal of Experimental Psychology, 1945, 35, 293-311.

PLATT, J. R. Discrete-trials and their relation to free-behavior situations. In $\mathbf{J}$. T. Spence and $H$. H. Kendler (Eds.) Essays in neobehaviorism: $A$ memorial volume to Kenneth $W$. Spence. New York: Appleton-Century-Crofts, in press

PORTER, J. J., \& HUG, J.J. Effects of number and percentage of rewarded trials on the acquisition and extinction of lever pressing using a descrete-trial procedure. Journal of Experimental Psychology $1965,70,575-579$. 